\title{
Editorial zum Themenschwerpunkt Wachstumslenkende Operation bei juvenilen Skoliosen
}

Eine operative Behandlung der Skoliose mit einer Versteifung ist bei einer Krümmung in der Brustwirbelsäule von $50^{\circ}$ und in der Lendenwirbelsäule von $45^{\circ}$ nach Cobb oder noch zu erwartender Progredienz indiziert. Die Patienten sollten jedoch das 12. Lebensjahr und mindestens das Skelettstadium Risser II erreicht haben. Sind eine dorsale Aufrichtung und dorsale Stabilisierung vor dem 10. Lebensjahr oder bei einem Risser $<$ II (Risser 0 oder Risser I) notwendig, kann es zu einem sog. Crankshaft-Phänomen kommen: Wegen des weiteren Wachstums der ventralen Wirbelsäulenabschnitte nehmen Skoliosewinkel, Rotation und Hyperlordosierung der versteiften Wirbelsäulenabschnitte erheblich $\mathrm{zu}$.

Unter einer juvenilen Skoliose versteht man eine frühkindliche Skoliose, die nicht erst mit Erreichen der Pubertät, sondern schon vor dem 5. Lebensjahr auftritt. Die juvenile Skoliose zeichnet sich durch eine meist rasche, konservativ nicht zu beeinflussende Progredienz bei gleichzeitiger Rigidität der Krümmung aus. Durch eine ausschließliche Korsettbehandlung in Kombination mit physikalischer Therapie ist das Fortschreiten nicht aufzuhalten. In diesen Fällen ist eine operative Behandlung schon im frühen Kindesalter erforderlich.

In diesem Schwerpunktheft werden die operativen Versorgungsstrategien mit unterschiedlichen Methoden in Abhängigkeit vom Kindesalter beschrieben. Ziel dieser Publikationen ist es, dem bereits versierten und interessierten Wirbelsäulenchirurgen neben den allgemeinen Beschreibungen der Operationstechnik praktische Hinweise und Tricks zur spezifischen operativen Versorgungsproblematik zu bieten.

Im ersten Beitrag wird der ventrale Zugang zur Wirbelsäule mit dem Halm-Zielke-Instrumentarium beschrieben. Die skoliotische Deformität wird von ventral dreidimensional korrigiert, sowohl in der Koronal- als auch Sagittalebene mit Schaffung eines physiologischen Wirbelsäulenprofils bei möglichst kurzer Fusionsstrecke. Die Indikation zur selektiven anterioren Korrektur der idiopathischen Skoliose kann bereits bei Risser I beginnen, da die ventralen Wachstumsfugen im zu fusionierenden Bereich zerstört werden. Nach der Lenke-Klassifikation eignen sich für ein solches Verfahren vor allem der Typ I (thorakal) und der Typ V (thorakolumbar/lumbar). Mit diesem operativen Ver- fahren ist keine Wachstumslenkung möglich. Es wird frühzeitig versteift.

Der zweite Beitrag befasst sich mit der operativen Behandlung der juvenilen Skoliose mit einem dorsalen Verfahren mit Pedikelschrauben und einer Wachstumslenkung. Die korrigierenden Stabilisierungsverfahren mit Fusion verhindern ein weiteres Wachstum. Durch die Implantation des StarLock-Instrumentariums oder eines anderen Schrauben-Stab-Systems in der Größe des StarLock-Instrumentariums wird ein korrigierendes Wirbelsäulenwachstum ermöglicht. Hierzu werden uni- oder bilateral zwei Stäbe verwendet, die proximal bzw. distal mit Pedikelschrauben an der Wirbelsäule verankert und über Parallelverbinder verschraubt werden. Das korrigierende Wachstum der Wirbelsäule wird durch ein- bis zweimaliges Nachspannen im Jahr erreicht. Dieses Operationsverfahren findet auch bei der neurogenen frühkindlichen Skoliose Anwendung.

Der dritte Beitrag beschäftigt sich mit der wachstumslenkenden Operation bei Kindern, die für ein StarLock- oder VEPTR-Instrumentarium zu groß sind. Das USS paediatric wurde speziell für die Indikation bei kleinen Kindern entwickelt und zeichnet sich durch ein niedriges Profil aus. Die Pedikelschrauben werden proximal und distal im Bereich der Neutralwirbel gesetzt und via den transmuralen Zugang nach Wiltse mit zwei Stäben mit Parallelverbindern verbunden. Der große Vorteil liegt in der transmuralen Einlage der Stäbe. In früheren Berichten brachte man den Stab subkutan ein (subkutaner Harrington). Dies führte gehäuft zu Wundheilungsstörungen. Die Indikation für ein wachstumslenkendes Operationsverfahren an der Wirbelsäule über 2-3 Jahre muss ausführlich mit den Patienten und Eltern besprochen werden, da man bei Kindern mit Risser I eine ventrale Epiphyseodese und dorsale Korrektur mit einem Pedikel/Stab-Instrumentarium kombinieren kann. Der Vorteil einer Wachstumslenkung liegt in einer Größenzunahme um 4-6 cm im Vergleich zu einer vorgezogenen Fusion.

Im vierten Beitrag wird die operative Behandlung von Skoliosen mit dem VEPTR-Instrumentarium dar-

Oper Orthop Traumatol 2010;22:121-2

DOI 10.1007/s00064-010-5002-3 
gestellt. Mit dem VEPTR-Instrumentarium werden das Thoraxvolumen erweitert, die Thoraxsymmetrie wiederhergestellt und die Lungenfunktion verbessert. Die Korrektur der Skoliose erfolgt nicht direkt durch eine Korrektur der einzelnen Wirbel, die instrumentiert werden, sondern über den Rippenthorax. Es ist keine direkte Präparation der Brustwirbelsäule notwendig. Ein Nachspannen findet alle 4-6 Monate statt.
Wir wünschen dem Leser eine interessante Lektüre und hoffen, dass die einzelnen Beiträge dem erfahrenen Chirurgen eine nützliche Hilfestellung bei der Indikationsstellung und operativen Versorgung der juvenilen Skoliose bieten.

Cornelius Wimmer 